\title{
Modelagem de Propriedades Físicas e Mecânicas em Painéis Aglomerados de Cedro Australiano
}

\author{
Lina Bufalino, Thiago de Paula Protásio, Antônia Amanda da Silva César, \\ Vânia Aparecida de Sá, Lourival Marin Mendes
}

Departamento de Ciências Florestais - DCF, Universidade Federal de Lavras - UFLA

\begin{abstract}
RESUMO
O objetivo deste trabalho foi avaliar a influência da razão de compactação sobre propriedades físicas e mecânicas de painéis aglomerados por meio de modelos lineares simples. Foram produzidos painéis em três composições distintas: 1) 100\% Toona ciliata; 2) 50\% Toona ciliata $+50 \%$ Pinus sp.; 3) 50\% Toona ciliata $+50 \%$ Eucalyptus sp. Para os modelos ajustados, foram realizadas análises de variância e teste de falta de ajuste. Os modelos foram avaliados de acordo com a significância de seus coeficientes, erro padrão da estimativa e coeficiente de determinação. A correlação linear de Pearson entre as variáveis foi determinada. Os módulos de elasticidade e ruptura aumentaram com o aumento da razão de compactação, tendo o contrário ocorrido para ligação interna, inchamento em espessura após 2 horas e absorção de água. Com exceção da compressão paralela às faces e inchamento em espessura, foram obtidos bons ajustes de modelos para as demais variáveis.
\end{abstract}

Palavras-chave: razão de compactação, modelos lineares, correlação.

\section{Modeling of Physical and Mechanical Properties of Australian Red Cedar Particleboards}

\begin{abstract}
The purpose of this research was to evaluate the influence of compression ratio on the physical and mechanical properties of particleboards through simple linear models. Panels were produced in three compositions: 1) $100 \%$ Toona ciliata; 2) 50\% Toona ciliata $+50 \%$ Pinus sp.; and 3) 50\% Toona ciliata $+50 \%$ Eucalyptus sp. For the adjusted models, variance analysis and lack of adjustment test were performed. The models were evaluated according to the significance of their coefficients, estimation standard error and determination coefficient. The Pearson linear correlation between variables was also determined. The elasticity and rupture modulus increased with the increase in compression ratio; the opposite occurred for internal bonding, thickness swelling after 2 hours and water absorption. Except for parallel to face compression and thickness swelling, good adjustments of models were obtained for the other variables.
\end{abstract}

Keywords: compression ratio, linear models, correlations. 


\section{INTRODUÇÃO}

A cultura das espécies dos gêneros Eucalyptus e Pinus para o suprimento das indústrias madeireiras de países tropicais é de suma importância para o abastecimento do setor madeireiro. No entanto, estudos para avaliar a potencialidade de produção de novas espécies florestais, bem como suas possibilidades de usos e aplicações, são importantes para diversificar a oferta de produtos desses países, aumentando sua competitividade no mercado global.

Dentre as espécies alternativas potenciais, o cedro australiano (Toona ciliata var. australis) vem se destacando em países como Brasil, Argentina, Havaí, Porto Rico, Honduras e outros. A Toona ciliata é uma espécie nativa que ocorre desde a Índia até a Austrália e que apenas há 200 anos crescia em grande abundância nesta região, mas que, devido à exploração do homem, teve grande parte de sua área destruída (Bygrave \& Bygrave, 2005). Esta espécie exótica adaptou-se muito bem as condições edafoclimáticas brasileiras, apresentando alta taxa de crescimento, madeira de boa qualidade e alta resistência ao ataque da mariposa de ponta (Hypsipyla grandella), importante praga que afeta os cedros nativos e o mogno brasileiro (Bygrave \& Bygrave, 2005; Pinheiro et al., 2006).

A madeira de cedro australiano, por ser considerada de alta qualidade, deve ser destinada preferencialmente à produção de madeira serrada. No entanto, qualquer processamento mecânico da madeira é responsável pela geração de grande quantidade de resíduos, os quais podem ser destinados à produção de painéis reconstituídos (Sá et al., 2010).

Deve-se considerar, porém, a baixa densidade básica da madeira de cedro australiano (Sá et al., 2010), que pode resultar em painéis aglomerados com razões de compactação acima da faixa ideal. Esta propriedade é definida pela razão entre a densidade do painel e a densidade da madeira, sendo intimamente relacionada com suas propriedades físicas e mecânicas. Altas razões de compactação normalmente estão relacionadas com alto desempenho mecânico, mas a absorção de água do ambiente pelo painel e, consequentemente, sua instabilidade dimensional podem aumentar (Kelly, 1977; Vital et al., 1974).

Para amenizar esse problema, madeiras de maior densidade básica podem ser misturadas com madeiras de menor densidade básica, possibilitando a produção de painéis com razão de compactação dentro da faixa adequada. Dessa forma, a mistura de espécies na composição de painéis aglomerados pode ser a resposta parcial para a utilização de espécies tropicais para essa finalidade (Moslemi, 1974; Vital et al., 1974).

$\mathrm{Na}$ literatura, podem ser encontrados estudos sobre o uso da modelagem estatística para avaliação da qualidade de painéis aglomerados. Essa técnica foi utilizada para relacionar teor de adesivo (Samin et al., 2010), proporção de madeira em mistura com resíduos (Arruda et al., 2011) e temperatura de prensagem (Silva et al., 2006) com propriedades físicas e mecânicas.

Diante do exposto, o objetivo deste trabalho foi avaliar a influência da razão de compactação sobre a qualidade física e mecânica de painéis aglomerados produzidos com diferentes combinações de madeiras por meio de modelos lineares simples.

\section{MATERIAL E MÉTODOS}

Foram utilizados resíduos do desdobro de toras (costaneiras) de madeira de Toona ciliata M. Roem var.australis (18 anos; densidadebásica $=0,320 \mathrm{~g} / \mathrm{cm}^{3}$ ), procedente do município de Marechal Floriano - ES; tábuas de madeira de Pinus sp. (18 anos; densidade básica $\left.=0,360 \mathrm{~g} / \mathrm{cm}^{3}\right)$, procedentes do plantio experimental do campus da Universidade Federal de Lavras - UFLA, MG; e tábuas de madeira de Eucalyptus sp. (7 anos; densidade básica $=0,530 \mathrm{~g} / \mathrm{cm}^{3}$ ), procedentes da Companhia Mineira de Metais, localizada no município de Vazante - MG. As tábuas e costaneiras foram inicialmente seccionadas em peças de $20 \mathrm{~cm}$ de comprimento, $9 \mathrm{~cm}$ de largura e 2,5 $\mathrm{cm}$ de espessura e, posteriormente, transformadas em partículas do tipo sliver por redução primária no picador de disco seguida de redução secundária no moinho martelo, utilizando peneira de $6,14 \mathrm{~mm}$. O material foi seco à temperatura de $103 \pm 2{ }^{\circ} \mathrm{C}$ até atingir a umidade base massa seca de aproximadamente $3 \%$ 
e, posteriormente, classificado em peneira vibratória para a remoção de "finos".

Para a manufatura dos painéis, foi utilizado o adesivo ureia-formaldeido a $9 \%$ com viscosidade de 618,94 cP, teor de sólidos de 56,78\% e pH de 7,5, além de emulsão de parafina na proporção de 1,5\%. $\mathrm{O}$ adesivo e a parafina foram aplicados sobre as partículas por meio de aspersão, utilizando pistola e homogeneizados em uma encoladeira do tipo tambor rotatório.

Os painéis foram produzidos em uma caixa formadora com as seguintes dimensões: comprimento de $480 \mathrm{~mm}$, largura de $480 \mathrm{~mm}$ e espessura de $15 \mathrm{~mm}$. A pré-prensagem foi feita em uma prensa manual, enquanto que a prensagem foi feita em um prensa hidráulica com controle de temperatura e pressão. As variáveis do ciclo de prensagem foram: temperatura de $160^{\circ} \mathrm{C}$, pressão de $40 \mathrm{kgf} / \mathrm{cm}^{2}$ e tempo de 8 minutos. Foram comparadas três composições diferentes dos painéis: 1) 100\% Toona ciliata; 2) 50\% Toona ciliata + 50\% Pinus sp.; 3) $50 \%$ Toona ciliata $+50 \%$ Eucalyptus sp. Foram produzidas três repetições por tratamento.

As seguintes propriedades físicas e mecânicas foram avaliadas: absorção de água e inchamento em espessura após 2 e 24 horas, módulo de elasticidade e módulo de ruptura em flexão estática, compressão paralela às faces e ligação interna. As propriedades físicas, ligação interna e compressão paralela foram determinadas de acordo com a norma ASTM D 1037-100 (ASTM, 2006), enquanto que a flexão estática foi realizada de acordo com a norma DIN 52362 (DIN, 1982). A densidade aparente e sua respectiva razão de compactação foram determinadas para cada corpo de prova.

Foram ajustados modelos lineares simples entre a razão de compactação e as propriedades físicas e mecânicas considerando todos os tratamentos, para os quais foram realizadas análises de variância e o teste de falta de ajuste, ambos a 5\% de significância. Os melhores modelos foram selecionados com base nos seguintes critérios: significância de todos os seus coeficientes (teste $t$ a $5 \%$ de significância); erro padrão da estimativa em porcentagem; e coeficiente de determinação.

Também foi avaliada a correlação linear de Pearson entre as variáveis dos modelos propostos, a qual foi analisada pelo teste $t$ a $5 \%$ de significância para verificar se o coeficiente de correlação é igual a zero e, consequentemente, avaliar a existência de uma relação linear entre as variáveis.

Todas as análises estatísticas foram efetuadas utilizando-se o software estatístico R versão 2.11.0, pacote stats (R Development Core Team, 2008).

\section{RESULTADOS E DISCUSSÃO}

O teste de falta de ajuste evidenciou que os modelos lineares se ajustaram bem aos dados, com exceção do modelo que relaciona razão de compactação e ligação interna. Na Tabela 1, estão apresentados os coeficientes ajustados e os parâmetros estatísticos utilizados para avaliação dos modelos lineares simples.

Os coeficientes de correlação lineares de Pearson foram não significativos $(\mathrm{p}>0,05)$ para as relações entre razão de compactação e inchamento em espessura após 2 horas de imersão e compressão paralela às faces. De forma análoga, a análise de variância evidenciou que os modelos que relacionam essas variáveis foram não significativos. Dessa forma, não existe relação linear entre a razão de compactação e as referidas variáveis (Figura 1).

Para as demais relações, foram encontrados coeficientes de correlação estatisticamente significativos. Foi observado que os coeficientes $\beta$ o e $\beta 1$ também foram significativos pelo teste $t(\mathrm{p}<0,05)$, com exceção do modelo ajustado entre o módulo de ruptura e razão de compactação, que apresentou $\beta o$ não significativo. Dessa forma, esse parâmetro pode ser retirado do modelo, contribuindo para melhorar a qualidade do ajuste. No entanto, esse modelo apresentou o menor erro padrão da estimativa.

Os maiores coeficientes de determinação foram encontrados para os modelos ajustados entre razão de compactação e a variável MOR, MOE e absorção de água após 24 horas de imersão. Dessa forma, nota-se a significativa porcentagem de variabilidade dessas variáveis que pode ser explicada pela razão de compactação.

Os maiores erros padrões da estimativa foram encontrados para os modelos ajustados entre a razão de compactação e o módulo de elasticidade e absorção de água após 2 horas de imersão. Os demais modelos 


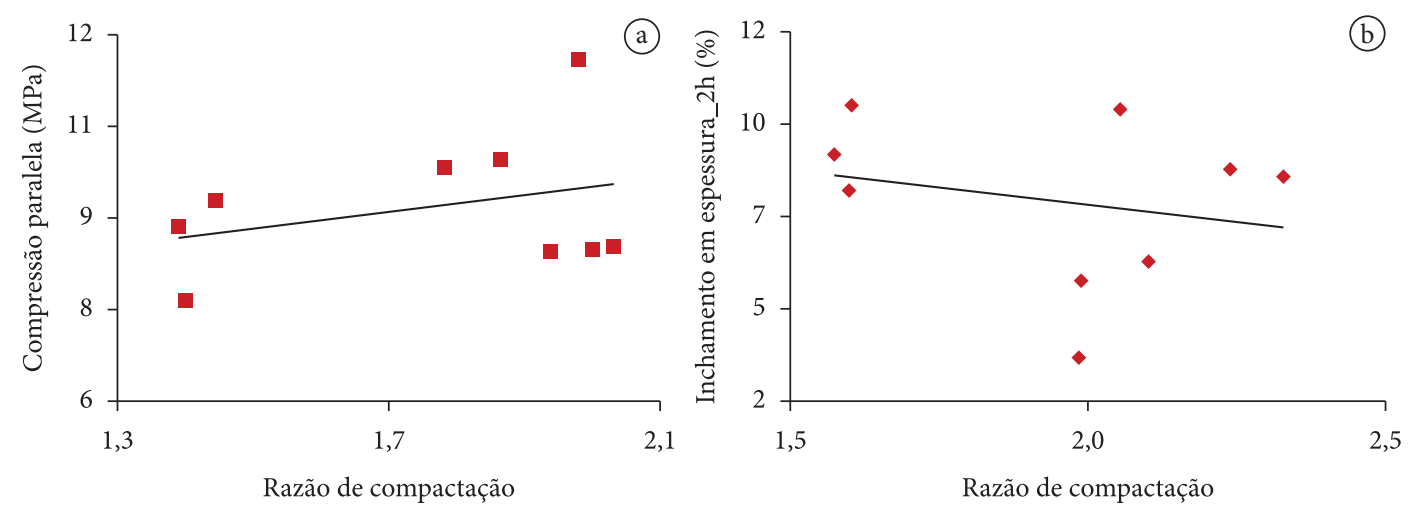

Figura 1. a) Relação entre razão de compactação e compressão paralela às faces; b) Relação entre razão de compactação e inchamento em espessura.

Figure 1. a) Relation between compression ratio and parallel to faces compression; b) Relation between compression ratio and thickness swelling.

Tabela 1. Parâmetros das regressões lineares simples ajustadas para a predição indireta das propriedades físicas e mecânicas.

Table 1. Parameters of the simple linear regressions adjusted for the indirect prediction of physical and mechanical properties.

\begin{tabular}{|c|c|c|c|c|c|c|c|}
\hline Modelo & \multicolumn{2}{|c|}{ Coeficientes } & tc & Fc & $\mathbf{R}^{2}$ & Syx (\%) & $\mathbf{r}$ \\
\hline \multirow{2}{*}{$A A \_2 h=f(R C)$} & $\beta_{0}$ & 35,353 & $4,382^{*}$ & \multirow{2}{*}{$9,34^{*}$} & \multirow{2}{*}{0,57} & \multirow{2}{*}{30,30} & \multirow{2}{*}{$-0,75^{\star}$} \\
\hline & $\beta_{1}$ & $-18,97$ & $-3,057^{\star}$ & & & & \\
\hline \multirow{2}{*}{$A A \_24 h=f(R C)$} & $\beta_{0}$ & 115,737 & $8,181^{*}$ & \multirow{2}{*}{$27,38^{*}$} & \multirow{2}{*}{0,79} & \multirow{2}{*}{13,68} & \multirow{2}{*}{$-0,89^{*}$} \\
\hline & $\beta_{1}$ & $-37,735$ & $-5,233^{*}$ & & & & \\
\hline \multirow{2}{*}{ IE_2h $=f(R C)$} & $\beta_{0}$ & 11,014 & $1,942^{\text {ns }}$ & \multirow{2}{*}{$0,3976^{\mathrm{ns}}$} & \multirow{2}{*}{0,05} & \multirow{2}{*}{31,58} & \multirow{2}{*}{$-0,23^{\mathrm{ns}}$} \\
\hline & $\beta_{1}$ & $-1,840$ & $-0,631^{\mathrm{ns}}$ & & & & \\
\hline \multirow{2}{*}{ IE_24h $=\mathrm{f}(\mathrm{RC})$} & $\beta_{0}$ & 40,168 & $6,869^{*}$ & \multirow{2}{*}{$12,671^{*}$} & \multirow{2}{*}{0,64} & \multirow{2}{*}{12,27} & \multirow{2}{*}{$-0,80^{*}$} \\
\hline & $\beta_{1}$ & $-10,611$ & $-3,560^{\star}$ & & & & \\
\hline \multirow{2}{*}{$\mathrm{LI}=\mathrm{f}(\mathrm{RC})$} & $\beta_{0}$ & 1,5916 & $6,080^{*}$ & \multirow{2}{*}{$9,223^{*}$} & \multirow{2}{*}{0,57} & \multirow{2}{*}{12,73} & \multirow{2}{*}{$-0,75^{*}$} \\
\hline & $\beta_{1}$ & $-0,4134$ & $-3,037^{\star}$ & & & & \\
\hline \multirow{2}{*}{$\mathrm{CP}=\mathrm{f}(\mathrm{RC})$} & $\beta_{0}$ & 6,832 & $2,519^{*}$ & \multirow{2}{*}{$0,771^{\mathrm{ns}}$} & \multirow{2}{*}{0,09} & \multirow{2}{*}{12,77} & \multirow{2}{*}{$0,31^{\text {ns }}$} \\
\hline & $\beta_{1}$ & 1,340 & $0,878^{\mathrm{ns}}$ & & & & \\
\hline \multirow{2}{*}{$\mathrm{MOE}=\mathrm{f}(\mathrm{RC})$} & $\beta_{0}$ & -2511 & $-2,389^{\star}$ & \multirow{2}{*}{$16,601^{\star}$} & \multirow{2}{*}{0,70} & \multirow{2}{*}{29,92} & \multirow{2}{*}{$0,84^{*}$} \\
\hline & $\beta_{1}$ & 2282 & $4,074^{*}$ & & & & \\
\hline \multirow{2}{*}{$\mathrm{MOR}=\mathrm{f}(\mathrm{RC})$} & $\beta_{0}$ & 3,982 & $1,743^{\text {ns }}$ & 42) $736 *$ & 0.86 & 505 & $0.93 *$ \\
\hline & $\beta_{1}$ & 7,954 & $6,537^{\star}$ & $42, / 36$ & 0,80 & 5,95 & 0,93 \\
\hline
\end{tabular}

tc: $t$ calculado; Fc: F calculado com 7 graus de liberdade para o erro; $\mathrm{R}^{2}$ : coeficiente de determinação; Syx: erro padrão da estimativa; r: coeficiente de correlação; $\mathrm{e}^{*}$ : significativo a 5\%; $\mathrm{e}^{\text {ns: }}$ : não significativo a 5\%; RC: razão de compactação; AA_2h e AA_24h: absorção após 2 e 24 horas, respectivamente (\%); IE_2h e IE_24h: inchamento em espessura após 2 e 24 horas, respectivamente (\%); LI: ligação interna (MPa); CP: compressão paralela às faces (MPa); MOE: módulo de elasticidade (MPa); MOR: módulo de ruptura (MPa).

apresentaram baixos valores desse parâmetro, com destaque para o modelo ajustado com o módulo de ruptura, o que indica que os pontos observados estão próximos da reta média ajustada.

Os resultados encontrados evidenciam que $70 \%$ da variabilidade do módulo de elasticidade e $86 \%$ da variabilidade do módulo de ruptura foram explicadas pela razão de compactação, demonstrando a importância dessa variável de processamento nas propriedades de flexão estática dos painéis aglomerados de cedro australiano.

Os gráficos dos modelos ajustados significativos e dos pontos observados estão apresentados na Figura 2. 

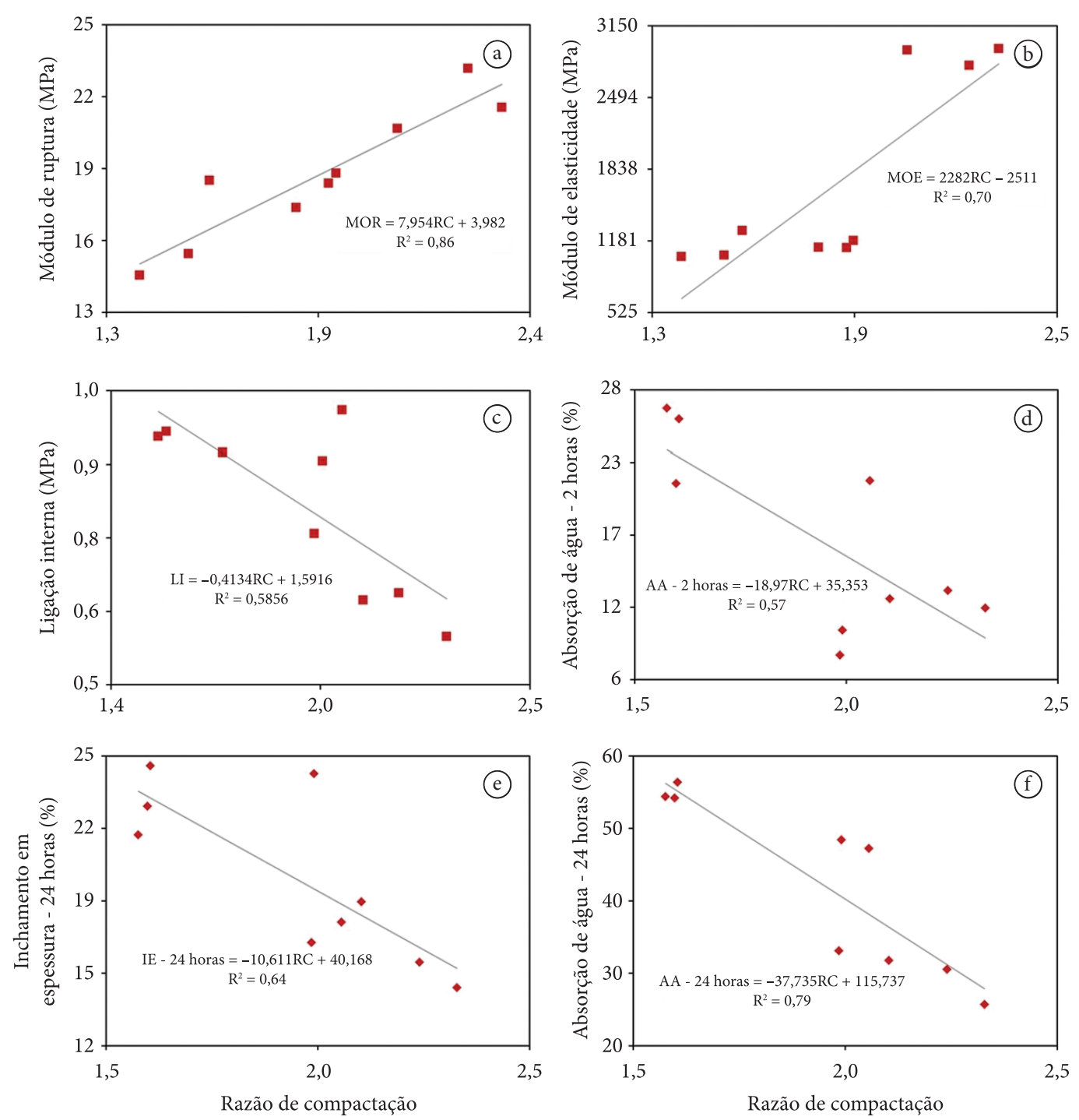

Figura 2. Relações encontradas entre razão de compactação e as seguintes propriedades: a) módulo de ruptura; b) módulo de elasticidade; c) ligação interna; d) absorção de água após 2 horas; e) inchamento em espessura após 24 horas; e f) absorção de água após 24 horas.

Figure 2. Relations found between compression ratio and the following properties: a) rupture modulus; b) elasticity modulus; c) internal bonding; d) water absorption after 2 hours; e) thickness swelling after 24 hours; and f) water absorption after 24 hours.

Foi observado que o aumento na razão de compactação ocasionou aumento das propriedades obtidas no teste de flexão estática. Analisando-se o coeficiente $\beta_{1}$ das equações, é possível observar a alteração esperada na variável dependente (y) quando a independente $(\mathrm{x})$ sofre uma variação unitária. Dessa forma, observou-se que o aumento de uma unidade na razão de compactação ocasiona aumento de 2282 e 7,954 MPa para os módulos de elasticidade e ruptura, respectivamente. Esse resultado pode ser atribuído ao maior volume de partículas compactadas e à maior área de contato entre partículas em painéis aglomerados de maior razão de compactação, o que resulta em melhor desempenho na absorção de forças mecânicas externas (Kelly, 1977; Vital et al. 1974).

$\mathrm{O}$ acréscimo de uma unidade na razão de compactação resulta em decréscimo de 0,41 $\mathrm{MPa}$ na ligação interna dos painéis, sendo esse resultado desvantajoso para a mistura de espécies florestais. 
A menor disponibilidade de adesivo por partícula em painéis de maior razão de compactação pode ter ocasionado essa tendência. Uma possibilidade de melhorar esse resultado seria aumentar o teor de ureia-formaldeído nos painéis de maior razão de compactação compostos por $100 \%$ de Toona ciliata e $50 \%$ de Toona ciliata mistura com 50\% de Pinus sp.

Para todas as propriedades físicas, foi observado que o aumento da razão de compactação melhorou o desempenho dos painéis. O acréscimo de uma unidade de razão de compactação ocasiona diminuições consideráveis de 19, 10 e $38 \%$ nas propriedades absorção de água após 2 horas de imersão, inchamento em espessura após 24 horas de imersão e absorção de água após 24 horas de imersão.

A tendência observada para absorção de água contrasta com os resultados normalmente relatados na literatura. Usualmente, maiores razões de compactação de painéis aglomerados resultam em valores maiores de absorção de água em função da maior área específica de madeira disponível para ligação com a água (Iwakiri et al., 2004). No entanto, os resultados encontrados neste trabalho podem ser explicados pela barreira física à entrada de água causada pela diminuição dos espaços vazios entre as partículas nos painéis de maior razão de compactação.

Espera-se que aumentos na razão de compactação ocasionem aumentos em inchamento em espessura após 24 horas de imersão devido à liberação mais intensa das forças de compressão geradas durante a prensagem do painel. Entretanto, maior área superficial de madeira em uma mesma unidade de volume pode também resultar em adição de pontos de contato entre partículas, o que pode diminuir o inchamento em espessura (Gatchell et al., 1966).

\section{CONCLUSÕES}

A inclusão da madeira de Toona ciliata de menor densidade às madeiras de Pinus sp. e Eucalyptus sp. de maior densidade resultou em aumentos na razão de compactação do painel e melhora de todas as propriedades físicas e mecânicas, com exceção da ligação interna.

A análise dos modelos demonstrou que a compressão paralela às faces e o inchamento dos painéis não apresentam relação linear com a razão de compactação. Para as demais variáveis, foram obtidos bons ajustes para os modelos, com destaque para o módulo de ruptura, indicando que $86 \%$ da variabilidade dessa propriedade foi explicada pela razão de compactação.

\section{AGRADECIMENTOS}

Os autores agradecem ao CNPq, FAPEMIG e Capes o apoio financeiro a esta pesquisa.

\section{STATUS DA SUBMISSÃO}

Recebido: 24/08/2011

Aceito: 07/02/2012

Resumo publicado online: 22/03/2012

Artigo completo publicado: 30/06/2012

\section{AUTOR(ES) PARA CORRESPONDÊNCIA}

\section{Lina Bufalino}

Departamento de Ciências Florestais - DCF, Universidade Federal de Lavras - UFLA, Campus Universitário, CEP 37200-000, Lavras, MG, Brasil e-mail: linabufalino@yahoo.com.br

\section{Thiago de Paula Protásio}

Departamento de Ciências Florestais - DCF, Universidade Federal de Lavras - UFLA, Campus Universitário, CEP 37200-000, Lavras, MG, Brasil e-mail: depaulaprotasio@gmail.com

\section{APOIO FINANCEIRO}

Capes, FAPEMIG, CNPq.

\section{REFERÊNCIAS}

American Society for Testing and Materials - ASTM. ASTM D1037-06a: standard test methods for evaluating properties of wood-base fiber and panel materials. Philladelphia: ASTM; 2006.

Arruda LM, Del Menezzi CHS, Teixeira DE. Lignocellosic composites from Brazilian giant bamboo (Guadua magna) Part 1: Properties of resin bonded particle boards. Maderas-Ciencia $Y$ Tecnología 2011; 13(1): 49-58. 
Bygrave FL, Bygrave PL. Growing Australian Red Cedar. Canberra: RIRDC/Land \& Water Australia/FWPRDC/ MDBC Joint Venture Agroforestry Program; 2005.

Gatchell GJ, Heebink BG, Hefty FV. Influence of component variables on the properties of particleboards for exterior use. Forest Products Journal 1996; 16(4): 45-59.

Iwakiri S, Shimizu J, Silva JC, Del Menezzi CHS, Puehingher CA, Venson ILC. Particleboard manufacturing from Grevillea robusta A. Cunn. ex R. Br. Revista Árvore 2004; 28(6): 883-887.

Kelly MW. Critical literature review of relationships between processing parameters and physical properties of particleboards. Madison: Forest Products Laboratory; 1977.

Moslemi AA. Particleboard. Carbondale: Southern Illinois University; 1974.

Deutsches Institut Für Normung - DIN. DIN 52362: Testing of wood chipboards bending test, determination of bending strength. Berlin; 1982. Normen Fur Holz Faserplaten Spanplatten Sperholz.

Pinheiro AL, Lani JL, Couto L. Cedro-Australiano: cultivo e utilização (Toona ciliata M. Roem. var. australis (F. Muell) Bahadur. Viçosa: UFV; 2006.
R Development core team. $R$ : A language and environment for statistical computing. Vienna: $\mathrm{R}$ Foundation for Statistical Computing; 2008. [cited 2012 jan. 8] Available from: http://www.R-project.org.

Sá VA, Mendes LM, Couto AM, Lima NN. Manufatura de painéis cimento-madeira de cedro australiano (Toona ciliata M. Roem var. australis) de diferentes procedências e idades. Scientia Forestalis 2010; 38(8): 559-566.

Samin Y, Ergun G, Mustafa C. The correlation of chemical characteristics and UF-Resin ratios to physical and mechanical properties of particleboard manufactured from vine prunings. Scientific Researches and Assays 2010; 5(8): 737-741.

Silva GA, Mendes LM, Trugilho PF, Mori FA, Santos IF, Pádua FA. Efeito de algumas variáveis de processamento nas propriedades físicas de painéis de madeira de partículas alongadas. Ciência Florestal 2006; 16(1): 51-60.

Vital BR, Lehmann B, Boone RS. How species and board densities affect properties of exotic hardwood particleboards. Forest Products Journal 1974; 24(12): 37-45. 